\title{
The economic evaluation of personalised oncology medicines: ethical challenges
}

\begin{abstract}
Jan $\mathbf{R} \mathbf{R}$ Lewis BPharm(Hons) PhD Candidate

Wendy L Lipworth MB BS, MSc, PhD Postdoctoral Fellow

Ian H Kerridge MPhil, FRACP, FRCPA

Director

Richard O Day MD, FRACP,

Professor of Clinical Pharmacology ${ }^{3}$

1 Centre for Values, Ethics and Law in Medicine, University of Sydney,

Sydney, NSW.

2 Australian Institute of Health Innovation, University of

New South Wales,

Sydney, NSW.

3 Clinical Pharmacology

and Toxicology,

St Vincent's Hospital,

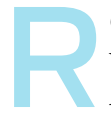
esearch in the post-genome era has revealed a variety of molecular drivers involved in cancer. Among these are mutations of the epidermal growth factor receptor gene (EGFR). Such mutations (EGFR $\mathrm{M}+$ ) are responsible for between $10 \%$ and $15 \%$ of advanced non-small cell lung cancer (NSCLC) cases in Australia.

Evidence indicates that less than a decade ago, the median survival after a diagnosis of advanced NSCLC was less than 12 months. At that time, randomised controlled trial (RCT) evidence indicated that platinum-containing chemotherapy was the most effective approach to disease management. ${ }^{1}$

More recent studies have shown that drugs targeting the receptor for epidermal growth factor (erlotinib and gefitinib) increase median survival of patients with EGFR $\mathrm{M}+$ advanced NSCLC to greater than 2 years - more than double previous survival rates. ${ }^{2}$

\section{Regulation and funding of EGFR tyrosine kinase inhibitors in Australia}

Sydney, NSW.

jlewis@

med.usyd.edu.au

doi: 10.5694/mjal3.10046

It is common for oncology treatments to first gain regulatory approval for the treatment of advanced disease; subsequent approval for use in earlier stages of disease is gained once sufficient evidence of effectiveness and safety becomes available.

Gefitinib and erlotinib were approved in 2004 and 2006, respectively, for the management of NSCLC patients who had exhausted other chemotherapy treatment options. Since then, gefitinib has been restricted to the management of EGFR M+ NSCLC only, while erlotinib may be prescribed for certain patients without the need for a genetic test.

Since their initial regulatory approval, evidence has emerged suggesting that erlotinib and gefitinib may be more effective and safer first-line treatments for EGFR M+ NSCLC than chemotherapy. ${ }^{3-8}$ For example, the Iressa Pan-Asia Study (IPASS) showed a significantly longer time to disease progression (9.5 versus 6.3 months; $P<0.001$ ) among patients with advanced EGFR M+ NSCLC treated with gefitinib compared with those who received first-line platinum-containing chemotherapy. In addition, significantly more patients treated with gefitinib had objective tumour shrinkage and experienced a better quality of life with fewer serious adverse events compared with those treated with chemotherapy. ${ }^{3}$

Additional evidence from non-randomised controlled studies suggested that patients with EGFR M+ NSCLC live longer when they receive gefitinib treatment than those who do not, ${ }^{9}$ and that EGFR M+ status by itself is not a significant prognostic predictor for better overall survival among patients treated with chemotherapy alone. ${ }^{10}$

\section{Summary \\ - Insights into the molecular drivers of cancer are providing opportunities for the development of new targeted treatments and more personalised approaches to cancer management \\ - Drugs targeting mutant epidermal growth factor receptors, such as erlotinib and gefitinib, may provide more effective, safer and better tolerated treatment options compared with chemotherapy among appropriately selected patients with advanced non- small cell lung cancer (NSCLC). \\ - First-line access to these newer treatments remains unfunded after several considerations by the Pharmaceutical Benefits Advisory Committee and their assessment that these are not cost-effective treatments. \\ - We suggest that there may be evidentiary and ethical challenges associated with the assessment of the cost- effectiveness of personalised oncology medicines in Australia, and that a new approach is needed to determine the value and cost-effectiveness of personalised medicine.}

Currently, the Pharmaceutical Benefits Advisory Committee (PBAC) has deemed this evidence insufficient to justify funding of EGFR tyrosine kinase inhibitors (TKIs) for first-line treatment of mutation-positive NSCLC, owing to unacceptably high incremental costs. They also expressed concerns regarding the validity of subgroup analyses, the generalisability of data to Australian patients and a lack of evidence that these treatments prolonged patient survival. ${ }^{11,12}$ The issue of survival prolongation is important because health technology assessments factor this into calculations of the qualityadjusted life-years (QALYs) gained from new treatments that determine their overall cost-effectiveness. ${ }^{13}$

While the PBAC's decision may have been appropriate, it is debatable whether the existing evaluation framework helps promote personalised medicine and whether it is appropriate for the assessment of the cost-effectiveness of treatments used in small populations defined by a biomarker. There are two key challenges faced by those wishing to demonstrate the value of such personalised medicines.

\section{Challenge one: disruption to accepted models used for constructing clinical evidence}

RCTs are regarded as optimal for assessing the effectiveness of treatments because they account for confounding due to known and unknown biases. However, because RCTs provide information about representative samples of populations they are not necessarily well suited to personalised medicine, which is concerned with highly specific sub- 
populations or individuals. Therefore, aggregated data from molecularly heterogeneous populations, expressed as average or median results, may not provide sufficiently detailed evidence for making personalised medicine decisions.

Furthermore, emergent evidence that benefits are restricted to patient subgroups identifiable by a biomarker may limit the usefulness of evidence from earlier RCTs and from any systematic reviews and meta-analyses based on them. This is problematic if regulatory and funding decisions that were based on less detailed and aggregated data are superseded by evidence that benefits are restricted to specific subgroups.

In the case of erlotinib and gefitinib, the significance of gene mutations became apparent only after regulatory approval; the effect sizes observed in RCTs, on which the decisions were based, would have been sensitive to the number of participants who had an EGFR M+ status. The outcomes of two similar Phase III studies among patients with NSCLC (known as ISEL and BR21) provide a possible example of this problem, as one study delivered a positive result while the other did not. ${ }^{14,15}$ An explanation may be that the positive study contained a higher proportion of EGFR $\mathrm{M}+$ patients than the negative study.

Furthermore, at the time of these two trials, diagnostic testing for EGFR mutations was not as sophisticated as it is today, and substandard testing could have skewed understanding of which patients truly benefit from TKI therapy. For example, analysis with a low-sensitivity test may have erroneously identified some mutation-positive participants as mutation negative, and therefore given the (false) impression that some patients with (apparently) EGFR mutation-negative NSCLC were responsive to TKI treatment. Because of these issues, questions persist about which populations truly stand to benefit.

Ultimately, such questions can only be resolved by evidence from further RCTs in which randomisation, and possibly stratification, of patients is done on the basis of known genetic characteristics identified by very accurate testing methods. However, such approaches are practically challenging and expensive, especially where the biomarker in question is rare.

Retrospective subgroup analysis of retained samples may also shed some light on these questions. Therefore, the collection and preservation of good-quality human biopsies and blood samples for which consent is obtained appropriately and prospectively may help overcome such questions in the future and may have benefits beyond the clinical trial setting.

\section{Challenge two: confounding due to crossover}

Before making a recommendation, the PBAC has to judge whether the incremental costs associated with a new listing represent value for money from the point of view of the government and community. To determine this, sponsors are asked to quantify increases in health benefit and weigh them against increases in cost. The PBAC generally demands evidence from at least one Phase III RCT showing a benefit in terms of QALYs. ${ }^{13}$

Six Phase III first-line RCTs have shown slower disease progression, fewer serious adverse events, improved quality of life and better symptom control among EGFR M+ patients receiving EGFR TKI therapy versus first-line chemotherapy. However, each failed to show a statistically significant difference in overall survival between treatment arms according to intention-to-treat analyses. Thus, evidence has not supported the cost-effectiveness of TKIs for the first-line treatment of NSCLC.

However, there are reasons to believe that the design of these RCTs may have obscured underlying and potentially significant survival (and therefore also economic) benefits.

First, there was striking variation between the average life expectancies reported in each of the four gefitinib studies, despite similar progression-free responses among those patients who first received chemotherapy (Box). The second observation is that each study showed longer median overall survival than that observed previously, less than a decade ago, with platinum-containing chemotherapy. Both of these observations require explanation.

One possibility is that, by themselves, EGFR mutations confer significant survival benefits. ${ }^{19}$ However, more recent evidence does not support this, as patients respond similarly to chemotherapy, $3,5,20$ deteriorate rapidly once they fail to respond to chemotherapy ${ }^{7,20}$ and generally have a similar prognostic outlook regardless of EGFR status. ${ }^{9,10}$ A more plausible explanation, therefore, may be that the EGFR TKIs have contributed to the survival of these patients, but that this has been obscured owing to study-treatment crossover.

To illustrate this link further, during the IPASS trial, $64 \%$ of EGFR M+ NSCLC patients crossed over from first-line chemotherapy to gefitinib after disease progression. ${ }^{21}$ This was in contrast to a study in north-eastern Japan, called NEJ002, in which $95 \%$ of similarly managed patients crossed over. The median survival among the IPASS group was 5 months shorter (22 months versus 27 months) despite similar initial responses (measured as progressionfree survival) to first-line platinum-containing chemother-

\begin{tabular}{|c|c|c|c|c|c|c|c|}
\hline \multirow[b]{2}{*}{ Study (known as) } & \multirow[b]{2}{*}{$n$} & \multirow[b]{2}{*}{ EGFR TKI used } & \multicolumn{2}{|c|}{ Median PFS (months)* } & \multicolumn{2}{|c|}{ Median OS (months) ${ }^{\dagger}$} & \multirow[b]{2}{*}{ Crossover (chemotherapy to TKI) } \\
\hline & & & TKI & Chemotherapy & TKI & Chemotherapy & \\
\hline Mok et al ${ }^{3}$ (IPASS) & 261 & Gefitinib & 9.5 & 6.3 & 21.6 & 21.9 & $47 \%$ \\
\hline Han et al ${ }^{5}$ (First-SIGNAL) & 32 & Gefitinib & 8.0 & 6.3 & 27.2 & 25.6 & $75 \%$ \\
\hline Inoue et $\mathrm{al}^{16}$ (NEJOO2) & 224 & Gefitinib & 10.8 & 5.4 & 27.7 & 26.6 & $95 \%$ \\
\hline Mitsudomi et $\mathrm{al}^{17}$ (WJTOG3405) & 172 & Gefitinib & 9.2 & 6.3 & 36 & 39 & $91 \%$ \\
\hline Rosell et $\mathrm{al}^{7}$ (EURTAC) & 174 & Erlotinib & 9.7 & 5.2 & 19.3 & 19.5 & $76 \%$ \\
\hline Zhou et $\mathrm{al}^{18}$ (OPTIMAL) & 154 & Erlotinib & 13.7 & 4.6 & 22.7 & 28.9 & $64 \%$ \\
\hline
\end{tabular}

EGFR $=$ epidermal growth factor receptor. * Differences statistically significant. $\dagger$ Differences not statistically significant. 
apy among patients in both studies. ${ }^{16,21}$ This suggests a correlation between TKI exposure and prolonged survival that was not shown by either study alone.

Crossover may also explain the unexpected observation that patients allocated to chemotherapy in two other firstline randomised trials, OPTIMAL ${ }^{8}$ and WJTOG $3405^{22}$ appeared to live up to 6 months longer on average than those allocated to first receive an EGFR TKI.

This is possibly because protocol-defined criteria, leading to crossover after disease progression, may have resulted in patients who were assigned first to chemotherapy being exposed to EGFR TKI therapy for a longer period of time than those initially allocated to receive erlotinib or gefitinib. This reinforces the suggestion that a correlation between TKI exposure and overall survival exists and that individual studies that include crossover are not ideal for quantifying the survival benefit from targeted drugs.

Retrospective statistical methods such as the rank-preserving structural failure time ${ }^{23}$ and inverse probability-ofcensoring weighting ${ }^{24}$ have been used with some success to estimate survival by overcoming confounding due to crossover. However, these methods have been mostly applied retrospectively, and they are limited in their ability to robustly quantify survival gains.

The question then follows whether, in order to demonstrate cost-effectiveness, crossover should be avoided to allow accurate assessment of survival impact. The ethical issues raised by this are enormously challenging - a situation that is likely to continue while regulators and payers advocate that evidence for survival benefits is best provided through interpretation of results from individual RCTs and meta-analyses.

\section{Conclusion}

The development of erlotinib and gefitinib illustrates the limitations of privileging RCTs when trying to assess the benefits of targeted treatments. Revision of the current evidence-based medicine model for assessing the clinical and cost-effectiveness of personalised therapies is, therefore, critically important, both for the design of ethical studies and the promotion of opportunities for personalised medicine in the future. This is especially important with regard to qualifying and quantifying the survival impact of treatments, which is critical to determining the cost-effectiveness of expensive new treatments, but hindered by most RCT designs. Understanding the overall survival impact and cost-effectiveness of new treatments will therefore require both new methodologies and new approaches to interpreting evidence.

The Australian Government's new approach for evaluating personalised therapies via the assessment of multiple codependent components (eg, drugs and companion diagnostics) is a step in the right direction. ${ }^{25}$ However, there remains little consensus among stakeholders about the status of RCTs relative to other trial designs, how to determine the significance of molecular testing or the ethical and epistemological issues surrounding trial design, especially when this relates to the demonstration of overall survival.

A supplementary approach to the latter may be to use observational cohorts, perhaps drawn from cancer registries, to characterise the natural history of biomarker-defined cancers, and compare outcomes from these with outcomes associated with patients who have received targeted therapies, either in trials or in real-world clinical settings.

While a number of legal, ethical and technical issues need to be resolved, such approaches may at least help those making funding decisions to determine whether the benefits of personalised medicine are worth paying for.

Competing interests: Jan Lewis is employed full-time by AstraZeneca Australia as associate medical director of oncology and infection.

Provenance: Not commissioned; externally peer reviewed.

1 Wakelee H, Belani CP. Optimizing first-line treatment options for patients with advanced NSCLC. Oncologist 2005; 10 Suppl 3: 1-10.

2 Ohashi K, Maruvka YE, Michor F, Pao W. Epidermal growth factor receptor tyrosine kinase inhibitor-resistant disease. J Clin Oncol 2013; 31: 1070-1080.

3 Mok TS, Wu YL, Thongprasert S, et al. Gefitinib or carboplatin-paclitaxel in pulmonary adenocarcinoma. $N$ Engl J Med 2009; 361: 947-957.

4 Maemondo M, Inoue A, Kobayashi K, et al; North-East Japan Study Group. Gefitinib or chemotherapy for nonsmall-cell lung cancer with mutated EGFR. N Engl J Med 2010; 362: 2380-2388.

5 Han JY, Park K, Kim SW, et al. First-SIGNAL: first-line single-agent Iressa versus gemcitabine and cisplatin trial in never-smokers with adenocarcinoma of the lung. J Clin Oncol 2012; 30: 1122-1128.

6 Mitsudomi T, Morita S, Yatabe Y, et al; West Japan Oncology Group. Gefitinib versus cisplatin plus docetaxel in patients with non-small-cell lung cancer harbouring mutations of the epidermal growth factor receptor (WJTOG3405): an open label, randomised phase 3 trial. Lancet Oncol 2010; 11: 121-128.

7 Rosell R, Carcenery E, Gervais R, et al. Erlotinib versus standard chemotherapy as first-line treatment for European patients with advanced EGFR mutation-positive non-small-cell lung cancer (EURTAC): a multicentre, open-label, randomised phase 3 trial. Lancet Oncol 2012; 13: 239-246.

8 Zhou C, Wu YL, Chen G, et al. Erlotinib versus chemotherapy as first-line treatment for patients with advanced EGFR mutation-positive non-small-cell lung cancer (OPTIMAL, CTONG-0802): a multicentre, open-label, randomised, phase 3 study. Lancet Oncol 2011; 12: 735-742.

9 Takano T, Fukui T, Ohe Y, et al. EGFR mutations predict survival benefit from gefitinib in patients with advanced lung adenocarcinoma: a historical comparison of patients treated before and after gefitinib approval in Japan. J Clin Oncol 2008; 26: 5589-5595.

$10 \mathrm{Lim} \mathrm{KH}$, Huang MJ, Liu HC, et al. Lack of prognostic value of EGFR mutations in primary resected non-small cell lung cancer. Med Oncol 2007; 24: 388-393.

11 Australian Government Department of Health and Ageing. Erlotinib public summary document. Pharmaceutical Benefits Advisory Committee meeting, July 2012. http://www.pbs.gov.au/industry/listing/ elements/pbac-meetings/psd/2012-07/erlotinib.pdf (accessed Jul 2013).

12 Australian Government Department of Health and Ageing. Gefitinib Public Summary Document. Pharmaceutical Benefits Advisory Committee meeting, November 2012. http://www.pbs.gov.au/industry/ listing/elements/pbac-meetings/psd/2012-11/gefitinib.pdf (accessed Jul 2013).

13 Birkett DJ, Mitchell AS, McManus P. A cost-effectiveness approach to drug subsidy and pricing in Australia. Health Aff (Millwood) 2001; 20: 104-114.

14 Shepherd FA, Rodrigues Pereira J, Ciuleanu T, et al. Erlotinib in previously treated non-small-cell lung cancer. NEngl J Med 2005; 353: 123-132.

15 Thatcher N, Chang A, Parikh P, et al. Gefitinib plus best supportive care in previously treated patients with refractory advanced non-small-cell lung cancer: results from a randomised, placebo-controlled, multicentre study (Iressa Survival Evaluation in Lung Cancer). Lancet 2005; 366: 1527-1537.

16 Inoue A, Kobayashi K, Maemondo M, et al; North-East Japan Study Group. Updated overall survival results from a randomized phase III trial comparing gefitinib with carboplatin-paclitaxel for chemo-naive non-small cell lung cancer with sensitive EGFR gene mutations (NEJ002). Ann Oncol 2013; 24: 54-59.

17 Mitsudomi T, Morita S, Yatabe Y, et al. Updated overall survival results of WJTOG 3405, a randomized phase II trial comparing gefitinib with cisplatin plus docetaxel as the first-line treatment for patients with non-small cell lung cancer harboring mutations of the epidermal growth factor receptor. (ASCO annual meeting proceedings, post-meeting edition). J Clin Oncol 2012; 30 (15 suppl): abstract 7521.

18 Zhou C, Wu YL, Liu X, et al. Overall survival results from OPTIMAL (CTONG0802), a phase III trial of erlotinib versus carboplatin plus gemcitabine as first-line treatment for Chinese patients with EGFR mutation-positive advanced non-small cell lung cancer. (ASCO annual meeting proceedings, post-meeting edition). J Clin Oncol 2012; 30 (15 suppl): abstract 7520.

19 Shepherd FA, Tsao MS. Unraveling the mystery of prognostic and predictive factors in epidermal growth factor receptor therapy. J Clin Oncol 2006; 24: 1219-1220.

20 Douillard JY, Shepherd FA, Hirsch V, et al. Molecular predictors of outcome with gefitinib and docetaxel in previously treated non-small-cell lung cancer: data from the randomized phase III INTEREST trial. J Clin Oncol 2010; 28: 744-752

21 Gridelli C, De Marinis F, Di Maio M, et al. Gefitinib as first-line treatment for patients with advanced non-smallcell lung cancer with activating epidermal growth factor receptor mutation: review of the evidence. Lung Cancer 2011; 71: 249-257.

22 Kobayashi K, Hagiwara K. Epidermal growth factor receptor (EGFR) mutation and personalized therapy in advanced nonsmall cell lung cancer (NSCLC). Target Oncol 2013; 8: 27-33.

23 Korhonen $\mathrm{P}$, Zuber E, Branson $\mathrm{M}$, et al. Correcting overall survival for the impact of crossover via a rankpreserving structural failure time (RPSFT) model in the RECORD-1 trial of everolimus in metastatic renal-cell carcinoma. J Biopharm Stat 2012; 22: 1258-1271.

24 Rimawi M, Hilsenbeck SG. Making sense of clinical trial data: is inverse probability of censoring weighted analysis the answer to crossover bias? J Clin Oncol 2012; 30: 453-458.

25 Merlin T, Farah C, Schubert C, et al. Assessing personalized medicines in Australia: a national framework for reviewing codependent technologies. Med Decis Making 2013; 33: 333-342. 